\title{
A novel in vitro method to identify protein glycation inhibitors
}

\author{
D.C.R. Wijetunge ${ }^{1,2}$, H.K.I. Perera ${ }^{1}$ \\ ${ }^{1}$ Department of Biochemistry, Faculty of Medicine, University of Peradeniya, Sri Lanka, ${ }^{2}$ Postgraduate Institute of Science, University of \\ Peradeniya, Sri Lanka
}

\section{A B S T R A C T}

Background: Protein glycation generates advanced glycation end products (AGEs) which are implicated in the pathogenesis of chronic complications associated with diabetes. Identification of medicinal plants with protein glycation inhibitory potential will enhance the opportunity to delay or inhibit diabetic complications with minimum side effects. Techniques available to identify protein glycation inhibitors require expensive specialized equipment. Objective: Objective of this study was to develop a relatively simple in vitro method to identify the protein glycation inhibitory potential of compounds or medicinal plants. Methods: Bovine serum albumin (BSA) was incubated with different concentrations of glucose or fructose or ribose for 31 days at $\mathrm{pH}$ 7.4. Standard inhibitor aminoguanidine (AG) was used as a positive control. Effect on the BSA migration under different experimental conditions was compared using polyacrylamide gel electrophoresis under native conditions (PAGE). Murraya koenigii leaf extract was analyzed for its effect on protein glycation. Results: We demonstrated many aspects of protein glycation including the effect of sugar concentration, type of the sugar and incubation period on protein glycation using this comparatively simpler method, which was previously, demonstrated using more sophisticated and expensive equipment. Migration of the BSA band towards the anode was proportionate to the degree of protein glycation. Further, we were innovative in demonstrating the inhibitory effect of AG on protein glycation using PAGE. BSA migration was comparatively slower when AG was included in the presence of sugar, indicating its inhibitory effects. We also revealed the protein glycation inhibitory potential of Murraya koenigii leaf extract, which was greater than that of AG at the concentrations used in the study. Conclusion: We have developed a novel simple in vitro method using PAGE to identify inhibitors of protein glycation.

Key words: Protein glycation, AGE, glycation inhibitors, PAGE, Murraya koenigii

\section{INTRODUCTION}

\section{Protein glycation}

Protein glycation involves a complex series of non-enzymatic covalent chemical reactions that occur between the carbonyl group of reducing sugars and the amino group of proteins. ${ }^{1}$ Equilibrium of the initial reversible reaction of glycation is highly dependent on the substrate concentrations and incubation time. Hence, glycation is closely associated with hyperglycemia and proteins with longer half lives. Over a period of time glycation intermediates undergo a series of irreversible reactions through highly reactive dicarbonyl intermediates
http://nepjol.info/index.php/AJMS 
a major pathogenic link between hyperglycemia and the long-term complications of diabetes ${ }^{3}$ and a large body of evidence has accumulated in this regard, throughout the 1980s and 1990s. ${ }^{1}$ AGEs are known to accelerate microvasculopathies and macrovasculopathies observed in diabetes including nephropathy, retinopathy, neuropathy and atherosclerosis. ${ }^{3-6}$

During later events of glycation, most AGEs form inter or intra molecular cross-links. Cross-linking of extracellular matrix proteins, especially collagen and elastin, leads to increased vascular stiffness and diminished vascular compliance. ${ }^{7}$ AGEs contribute to the development of a pro-inflammatory and proliferative state with increased oxidative stress, resulting in endothelial dysfunction, the common underlying mechanism leading to organ damage. ${ }^{8}$

\section{Protein glycation inhibitors}

Inhibition of AGE formation is one of the therapeutic approaches that delay or prevent the progression of diabetic complications. ${ }^{9,10}$ Many studies showed that aminoguanidine (AG), an inhibitor of AGE formation studied extensively, is effective in preventing chronic diabetic complications. ${ }^{4}$ However, designing a drug having anti-AGE activity is a challenge due to the complexity of reactions involved in AGE formation. A number of compounds identified with potential glycation inhibitory effect including AG, failed at clinical trials due to their side-effects. ${ }^{4}$ Therefore, identification of new antiglycating agents with higher levels of efficacy and safety in humans is very much needed.

\section{Potential of medicinal plants in inhibiting protein} glycation

Humankind has a long history in the use of herbal medicines. Use of medicines of natural origin appears to offer more gentle means of managing diseases at a lower cost. Investigation of protein glycation inhibitors, especially natural agents with few side-effects, may yield a potential therapeutic approach for delaying and preventing diabetic complications. Recent studies have highlighted the benefits of using plants with antiglycation properties in diabetic patients. $^{9}$

Some plants have been investigated for protein glycation inhibitory activities using several protein-sugar models such as bovine serum albumin (BSA) and fructose. It is evident that a positive correlation exists between the total phenolic content of the plant extract and their glycation inhibitory potential. Further, purified phenolic compounds such as flavones, proanthocyanidins, and other phenolics were found to have a strong inhibitory activity. ${ }^{11}$ However, Povichit et al., found a strong antiglycating potential with some plant extracts having relatively low contents of total phenolic compounds. ${ }^{12}$ A good correlation has been shown to exist between free radical scavenging capacity of the plant extract and AGE inhibitory activity in vitro. ${ }^{11,13}$ Chen et al., suggested that the total saponin content too is correlated to AGE inhibitory potential. ${ }^{13}$ In a recent review, anti-glycation properties of some foods, their active components, and their mechanisms of action were discussed.

Analytical methods used to detect protein glycation and inhibition

Several assay methods have been used to determine the effect of glycation and inhibition, including assays based on measurement of Amadori products formed such as HbA1c, specific fluorescence generated in the course of AGE-formation and formation of protein crosslinks. ${ }^{2,10,14,15}$ High performance liquid chromatography, mass spectrometry, fluorescence spectrometry, specific ELISA assays and Western blotting methods using antiAGE antibodies have been used to detect the glycation products. In many of these methods BSA, haemoglobin, lysozyme and collagen were used as target proteins with different sugars such as glucose, fructose and ribose or more reactive dicarbonyl compounds such as methyl glyoxal used as glycating agents. $2,6,10,14-16$

Objective of this study was to develop a relatively simple in vitro method to identify the protein glycation inhibitory potential of compounds or medicinal plants.

\section{MATERIALS}

\section{Chemicals}

Bovine Serum Albumin, Aminoguanidine, D-glucose, D-fructose, D-ribose, $\mathrm{Na}_{2} \mathrm{HPO}_{4}, \mathrm{NaH}_{2} \mathrm{PO}_{4}$, Sodium azide, Tris, Glycine, TEMED, Ammonium per sulphate and Acrylamide were from Sigma.

Plant parts

Plant parts were obtained from Kandy, Sri Lanka.

\section{METHODS}

Following methods were repeated at least on four different occasions.

\section{In vitro protein glycation \\ Effect of different sugars and $A G$ on protein glycation}

BSA was incubated at $37^{\circ} \mathrm{C}$ in phosphate buffer (PB) at $\mathrm{pH} 7.4$ in sealed glass tubes, ${ }^{2}$ with varying concentrations of glucose, fructose and ribose $(0-500 \mathrm{mM})$ in the presence or absence of the standard glycation inhibitor AG $(10 \mathrm{mM})$. 


\section{Effect of different AG concentrations on protein glycation}

BSA was incubated at $37^{\circ} \mathrm{C}(\mathrm{pH} 7.4)$ with fructose $(500 \mathrm{mM})$ and different concentrations $(1,10,20 \& 40 \mathrm{mM})$ of AG.

\section{Effect of incubation temperature on protein glycation}

BSA was incubated at different temperatures $\left(27-50^{\circ} \mathrm{C}\right)$ in the presence of glucose and ribose. Results were compared with a control with no incubation.

\section{Effect of different sugars and AG on human serum protein glycation}

Human serum was incubated with $500 \mathrm{mM}$ glucose, fructose and ribose in the presence and absence of $10 \mathrm{mM} \mathrm{AG}$ for 7 days. BSA was incubated with fructose for comparison.

Aliquots of the incubated samples were collected at selected intervals for up to 31 days and stored at $-40^{\circ} \mathrm{C}$ for further analysis.

\section{DETECTION METHODS}

Aliquots were analyzed using polyacrylamide gel electrophoresis under non-denaturing and denaturing conditions. Changes in migration positions of the BSA bands were compared in non-glycated, glycated and inhibited reactions after staining the gel.

Polyacrylamide gel electrophoresis under native conditions (PAGE)

Polyacrylamide gels $(10 \%)$ were prepared according to the standard technique ${ }^{17}$ (Enduro Vertical Gel Electrophoresis system- E2010-P). Samples were loaded without denaturing the proteins. After separation at $\mathrm{pH}$ 8.6, protein bands were visualized by staining with Coomassie brilliant blue. Changes in the migration position of BSA bands were compared.

Sodium dodecyl sulphate polyacrylamide gel electrophoresis under denaturing conditions (SDS PAGE)

SDS-Polyacrylamide gels $(12 \%)$ were prepared according to the standard technique ${ }^{17}$ using the same electrophoresis system. Samples were loaded after heating with SDS sample buffer at $95^{\circ} \mathrm{C}$. Rest of the procedure was similar to that of PAGE.

A Novel method to identify protein glycation inhibitory potential of Murraya koenigii

Specimen of Murraya koenigii (L.) Spreng (Curry leaf in English) was authenticated at the National herbarium, Royal Botanical Gardens, Peradeniya, Sri Lanka.
Water extract of dried M. koenigii leaf was prepared. Glycation of BSA was undertaken using the optimized method. In brief, BSA was incubated with fructose $(500 \mathrm{mM})$ in $\mathrm{PB}(\mathrm{pH} 7.4)$ containing $0.02 \%$ sodium azide in the dark at $37^{\circ} \mathrm{C}$ for 30 days. These incubations were conducted in the presence or absence of the plant extract $(5 \mathrm{mg} / \mathrm{ml})$. AG $(10 \mathrm{mM})$ was used as the standard inhibitor. Corresponding blanks were prepared in the absence of fructose. Aliquots were collected at intervals up to 30 days and analyzed using PAGE (under non denaturing conditions) as described above.

\section{RESULTS}

Migration of the BSA band under non denaturing conditions (PAGE) towards the anode showed clearly detectable variation with the experimental conditions used. Increase in BSA migration seemed to be proportionate to the degree of protein glycation. BSA migration increased with increasing sugar concentration from 0 to $500 \mathrm{mM}$ (Figure 1) and duration of incubation from 0 to 5 days with $500 \mathrm{mM}$ sugar (Figure 2). Three sugars used showed a variation in the rate of protein modification during early incubation, where ribose showed the most rapid effect (Figure 2) while glucose showed the slowest (Figure 4-Left). With longer incubations the rate of BSA migration looked relatively similar with different sugars used (Figure 4-Right). Changes in the migration of human serum albumin in presence of the three sugars (500 $\mathrm{mM}$ ) were comparable with that of BSA (Figure 3). BSA migration increased with increasing incubation temperature from 27 to $50^{\circ} \mathrm{C}$ (Figure 5).

AG decreased BSA migration in the presence of sugar. There was no effect on BSA migration by AG in the absence of sugar (Figure 6). AG showed a dose-dependent inhibition of BSA migration from 1 to $10 \mathrm{mM}$ in the presence of $500 \mathrm{mM}$ sugar (Figure 7). A similar inhibition was not visible with an increase of 10 to $40 \mathrm{mM} \mathrm{AG}$ (Results not shown). AG (10 mM) also inhibited the migration of human serum albumin in the presence of $500 \mathrm{mM}$ sugar (Figure 3).

Migration of the BSA band under denaturing conditions (SDS-PAGE) also showed variation (but to a lesser degree

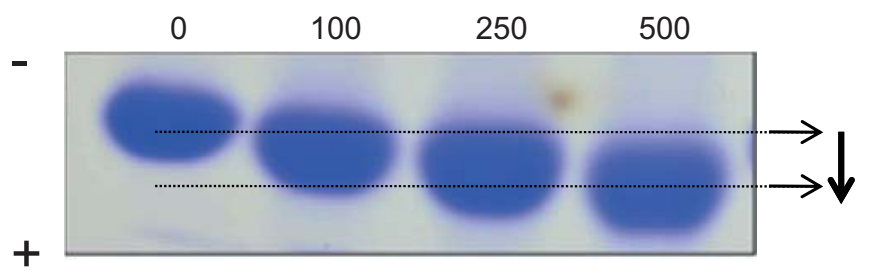

Figure 1: Effect of fructose concentration on BSA migration with PAGE. (Incubation period was 5 days. Arrow indicates the increase in BSA migration with the increase of sugar concentration from 0-500 mM.) 


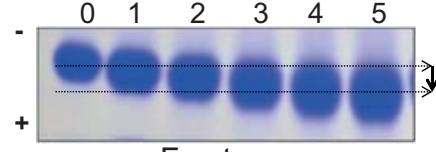

Fructose

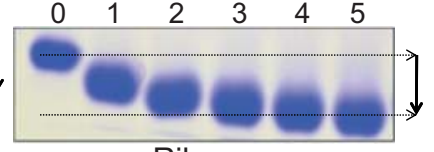

Ribose
Figure 2: Effect of different sugars and incubation period on BSA migration with PAGE (Incubation period was 0-5 days. 0 (Day 0): BSA with sugar without incubation. Arrows indicate the increase in BSA migration with the increase of incubation period. This increase was relatively higher in presence of ribose)

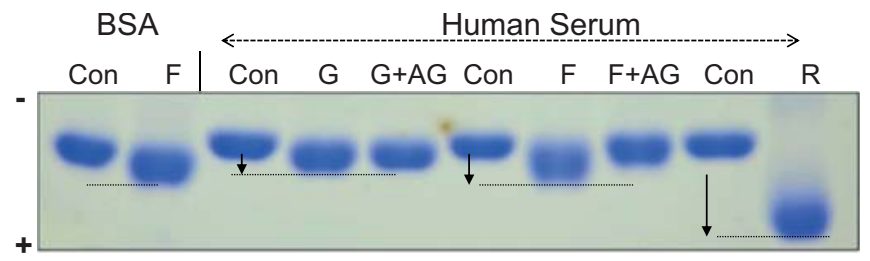

Figure 3: Effect of different sugars on migration of human serum albumin with PAGE (Incubation period was 7 days. G: Glucose, F: Fructose, R: Ribose, Con: BSA/ human serum with $\mathrm{PB},+\mathrm{AG}$ : in presence of $A G$. Human serum was used as the source of human serum albumin. Area representing the albumin band is shown. Arrows indicate the increase in migration of albumin bands in presence of sugar)

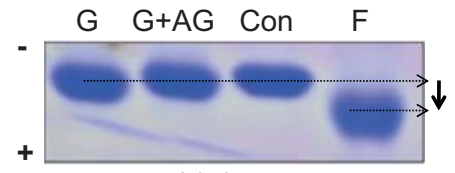

14 days

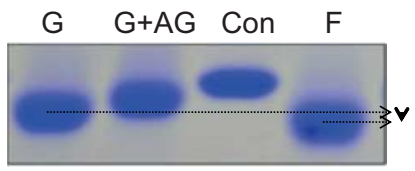

31 days
Figure 4: Effect of glucose and fructose on BSA migration at Day 14 (left) and Day 31 (right) incubations with PAGE (Con: Negative control: BSA with PB, G: BSA with Glucose, F: BSA with Fructose, G+AG: $B S A$ with glucose and $A G$. Arrows indicate the relative increase in BSA migration in presence of fructose, compared to that of glucose at day 14 and 31 )

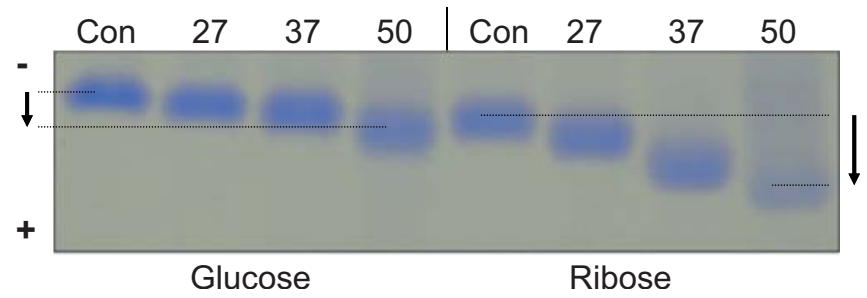

Figure 5: Effect of temperature in presence of glucose and ribose on BSA migration with PAGE. (Con: BSA and sugar without incubation. Samples were incubated at $27,37 \& 50^{\circ} \mathrm{C}$ for 3 days. Aliquots collected from different temperatures were loaded on the same gel. Arrows indicate the increase in BSA migration with increase in temperature. This increase was higher in presence of ribose.)

compared to that of PAGE) under the experimental conditions used, with a decrease in migration towards anode seemingly proportionate to the degree of protein glycation. A decrease in migration of the BSA band was observed with increasing incubation time with sugar (Figure 8) and increasing concentration of sugar (Figure 9) suggesting an increase in MW of BSA due to attachment of sugar molecules and other intermediates

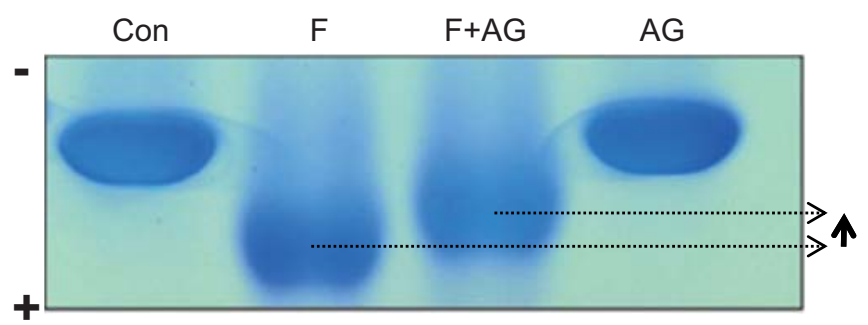

Figure 6: Effect of glycation inhibitor AG on BSA migration with PAGE. (Con: Negative control: BSA with $\mathrm{PB}, \mathrm{F}$ : BSA with Fructose, $\mathrm{F}+\mathrm{AG}$ : $B S A$ with Fructose and $A G, A G$ : BSA with $P B$ and $A G$. Arrow indicates the decrease in $B S A$ migration in presence of $A G$.)

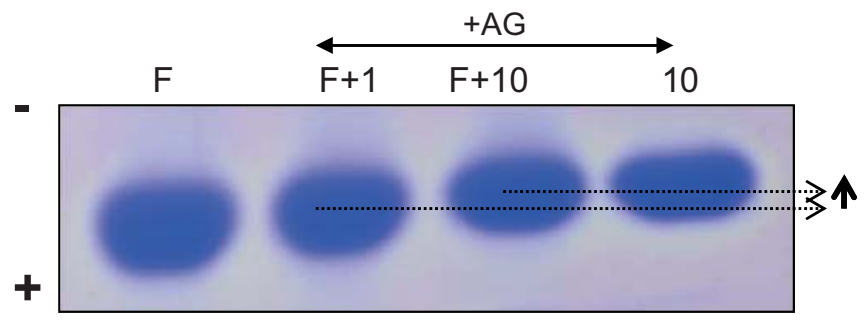

Figure 7: Dose-dependent inhibition on BSA migration in the presence of AG with PAGE. (F: BSA with Fructose, $F+1$ : BSA with Fructose and $1 \mathrm{mM} \mathrm{AG}, \mathrm{F}+10$ : BSA with Fructose and $10 \mathrm{mM} \mathrm{AG}, 10$ : BSA with $\mathrm{PB}$ and $10 \mathrm{mM}$ AG. Arrow indicates the decrease in BSA migration in presence of 1 and $10 \mathrm{mM} \mathrm{AG}$.)
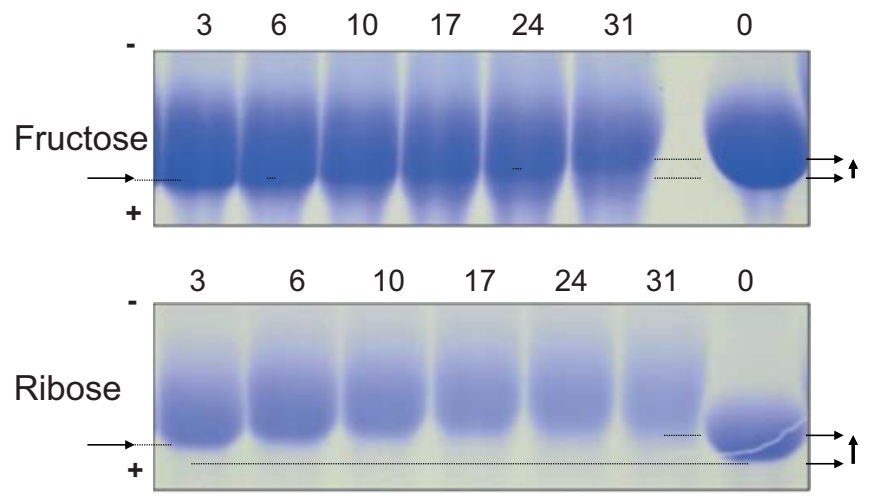

Figure 8: Effect of incubation period on BSA migration with SDS PAGE. (0 (Day 0): BSA without incubation. Incubation period was 0-31 days. Arrows indicate the decrease in BSA migration with increase in incubation period. This decrease was higher in presence of ribose.)

of glycation. A difference in the colour intensity within the BSA band was observed with fructose, suggesting that the degree of glycation is not uniform on all the BSA molecules present (Figure 8). Decrease in BSA migration was more marked in presence of ribose compared to that of fructose (Figure 8).

In the presence of Murraya koenigii leaf extract, migration of BSA under non denaturing conditions was reduced compared to the migration of BSA in absence of the extract, suggesting an in vitro protein glycation inhibitory potential of the leaf extract. This inhibition was greater than that of AG under the conditions used (Figure 10). 


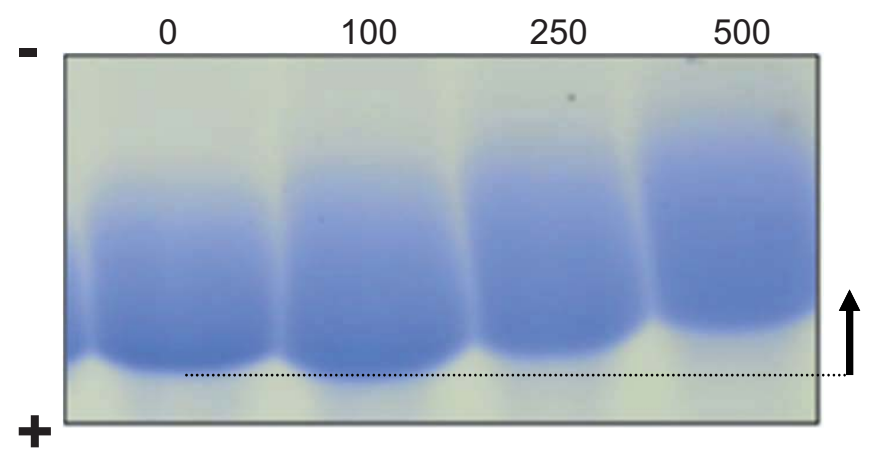

Figure 9: Effect of sugar concentration on BSA migration with SDS PAGE. (Incubation period was 7 days. Sugar concentrations used were $0-500 \mathrm{mM}$. Arrow indicates the decrease in BSA migration with increase in sugar concentration.)

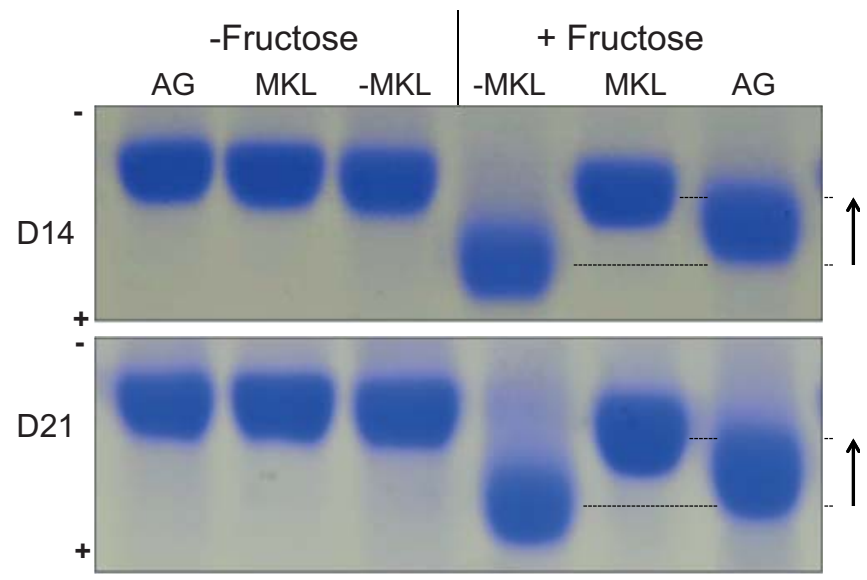

Figure 10: Effect of Murraya koenigii leaf extract on BSA migration with PAGE. (MKL: in presence of Murraya koenigii leaf extract, -MKL: in absence of Murraya koenigii leaf extract, AG: in presence of $A G$, -Fructose: BSA incubated without fructose, +Fructose: BSA incubated with Fructose. Incubation period was 14 (D14) and 21 (D21) days. Arrows indicate the decrease in BSA migration in presence of Murraya koenigii leaf extract at Day 14 and 21. This decrease was greater than that of $A G$.)

\section{DISCUSSION}

Glycation is identified as a key molecular mechanism of chronic diabetic complications. Identification of medicinal plants with protein glycation inhibitory potential will enhance the therapeutic strategies to delay or inhibit diabetic complications with minimum side effects. Analytical techniques available to identify protein glycation inhibitors require expensive specialized equipment. We have developed a novel simple technique which could be used to detect protein glycation inhibitory potential of medicinal plants.

Using a comparatively simpler PAGE method, we demonstrated many aspects of protein glycation, which were previously demonstrated only by using more sophisticated and expensive equipment. ${ }^{12,13,15,19}$ These include the effect of sugar concentration, type of the sugar, incubation period and incubation temperature on protein glycation. We observed changes in BSA migration with both PAGE and SDS-PAGE, which seemed to be proportionate to the extent of glycation.

Electrophoretic migration under native conditions allows evaluation of some modifications that alter either the charge or the conformation of a protein. Glycation affect the net charge of a protein as the positively charged free amino groups are targets of glycation, to which negatively charged reducing sugars are attached. In our study, BSA migration towards the anode in PAGE was increased with the conditions which speed up glycation such as increase in sugar concentration, incubation period and incubation temperature. Sauradipta et al., demonstrated that myoglobin glycated with highly reactive dicarbonyl intermediate methyl glyoxal exhibiting higher electrophoretic mobility in native PAGE compared to the native myoglobin..$^{20}$ Our results with relatively less reactive sugars were comparable to that of Sauradipta et al. ${ }^{20}$ Based on our findings and previous studies, we suggest that the observed increase in BSA migration towards the anode was proportionate to the extent of glycation of BSA.

We observed changes in the BSA migration upon glycation, with SDS-PAGE too but to a lesser degree. LedesmaOsuna et al., revealed similar findings with SDS PAGE of glycated BSA, where BSA bands migrated broader and slower than native BSA. Using mass spectrometry they demonstrated that glycated BSA contain a range of protein molecules with different numbers of coupled sugar residues. ${ }^{21}$ Our results which are in agreement with those of Ledesma-Osuna et al. ${ }^{21}$ suggest that the decrease in migration observed was due to the increase in molecular weight of BSA due to glycation.

Wei et al., demonstrated that ribose more rapidly glycates BSA among other sugars such as glucose, fructose, xylose and sucrose using several methods including fluorescence spectrophotometry and SDS PAGE. ${ }^{15}$ Meeprom et al. showed that the rate of glycation of BSA is much faster in presence of fructose compared to that of glucose. ${ }^{18}$ Our findings are similar to those of ribose, fructose and glucose on the rate of increase in net negative charge and molecular weight of BSA. Our observation on the effect of glucose at later stages which show speeding up of glycation could be a novel finding. Glucose is, known to be least reactive of the common sugars, perhaps leading to its evolutionary selection as the principal free sugar in vivo. ${ }^{1}$ We have also observed similar results using human serum as a source of human albumin which shows the possibility of applying the findings to human proteins as well. 
In our study, we were innovative by using the PAGE method under native conditions in identifying the inhibitory effect on protein glycation. AG which is known to trap the reactive carbonyl groups of glycation products, ${ }^{4}$ inhibited protein glycation in a dose-dependent manner, under the experimental conditions used in our study. We also showed similar inhibitory effect of AG on glycation of human serum albumin.

Furthermore Murraya koenigii leaf extract has showed an in vitro protein glycation inhibitory potential with the developed PAGE method revealing the feasibility of this method in identifying glycation inhibitory potential of medicinal plants. This inhibition was greater than that of AG at the concentrations used in the study. M. koenigii commonly known as curry leaf is used as a natural flavoring agent during preparation of food and for medicinal purposes. Several studies have shown antidiabetic activity of $M$. koenigii leaf including hypoglycaemic effects. ${ }^{22}$ Administration of leaf extract in to strpotozotocininduced diabetic rats demonstrated a protective effect against development of diabetic neuropathy and a decline in HbA1c level..$^{23}$ Antioxidant and free radical scavenging activities and nephroprotective effects of the leaf are also proven..$^{22}$ Hypoglycaemic effects of the leaf extract would contribute to a decline in protein glycation in vivo. Our results suggest an additional antiglycating effect, independent of hypoglycaemic effects of $M$. koenigii leaf, as the sugar concentrations used in the reactions were the same in the presence and absence of plant extract.

The method we developed can be described as a qualitative and a semi quantitative method which can identify relative differences in glycation inhibition. A limitation of this method over other established methods such as HPLC and fluorescence spectrophotometry is its difficulty in quantifying the degree of glycation inhibition precisely.

We reveal the capability of this novel PAGE based method, in detecting potential inhibitors of protein glycation, in the search of new natural therapeutics to delay or prevent diabetic complications. Currently we are investigating the glycation inhibitory potential of medicinal plants with known antidiabetic effects, using the developed method.

\section{CONCLUSION}

We have developed a novel in vitro method using PAGE to detect inhibitors of protein glycation. We have demonstrated protein glycation inhibitory potential of the M. koenigii leaf.

\section{ACKNOWLEDGEMENTS}

Financial assistance by the University of Peradeniya Research Grants, RG/2011/27/M and RG/2012/CG$1 / 38 / \mathrm{M}$.

Prof. R. Sivakanesan for revising the manuscript.

Mr. A.M.P.S.T.M. Bandara for technical assistance.

\section{REFERENCES}

1. Ulrich $P$ and Cerami $A$. Protein glycation, diabetes and aging. Recent Progress in Hormone Research 2001;56:1-22.

2. Gutiérrez RMP, Diaz SL, Reyes IC and Gonzalez AMN. Antiglycation effect of spices and chilies used in traditional Mexican cuisine. J Nat Prod 2010;3:95-102.

3. Jakus $V$ and Rietbrock N. Advanced glycation end-products and the progress of diabetic vascular complications. Physiol Res 2004;53:131-142.

4. Rahbar S and Figarola JL. Novel inhibitors of advance glycation end products. Arch Biochem Biophy 2003;419:63-79.

5. Wang $Y$, Vom Hagen F, Pfister F, Bierhaus A, Feng Y, Gans R, et al. Receptor for advanced glycation end product expression in experimental diabetic retinopathy. Ann N Y Acad Sci 2008; 126:42-45. doi: 10.1196/annals.1433.063.

6. Muthenna P, Akileshwari C and Reddy GB. Ellagic acid, a new antiglycating agent: its inhibition of $\mathrm{N} \varepsilon$-(carboxymethyl) lysine. Biochem J 2012;442:221-230.

7. Pathak P, Gupta R, Chaudhari A, Shiwalkar A, Dubey A, Mandhare $A B$, et al. A novel advanced glycation end product breaker TRC4149 improves hemodynamic status in diabetic spontaneously hypertensive rats. Eur J Med Res 2008;13: 388-398.

8. Giuseppina B, Ann Marie $S$ and Raffaele DC. Advanced glycation end products and vascular inflammation: implications for accelerated atherosclerosis in diabetes. Cardiovascular Research 2004;63: 582-592.

9. Wu C, Huang S, Lin J and Yen G. Inhibition of advanced glycation endproduct formation by foodstuffs. Food Funct 2011; 2:224-234.

10. Adisakwattana S, Sompong W, Meeprom A, Ngamukote S and Yibchok-anum S. Cinnamic acid and its derivatives inhibit fructosamine-mediated protein glycation. Int J Sci 2012; 13: 1778-1789.

11. Dearlove RP, Greenspan P, Hartle DK, Swanson RB, and Hargrove JL. Inhibition of protein glycation by extracts of culinary herbs and spices. J Med Food 2008;11(2):275-281.

12. Povichit $N$, Phrutivorapongkul $A$, Suttajit $M$, Chaiyasut $C$ and Leelapornpisid. P. Phenolic content and in vitro inhibitory effects on oxidation and protein glycation of some Thai medicinal plants. Pak J Pharm Sci 2010;23(4):403-408.

13. Chen Y-F, Roan H-Y, Lii C-K, Huang Y-C and Wang T-S. Relationship between antioxidant and antiglycation ability of saponins, polyphenols, and polysaccharides in Chinese herbal medicines used to treat diabetes J Medicinal Plants Research 2011;5(11):2322-2331.

14. Xi M, Hai C, Tang H, Chen M, Fang $\mathrm{K}$ and Liang $\mathrm{X}$. Antioxidant and antiglycation properties of total saponins extracted from traditional Chinese medicine used to treat diabetes mellitus. Phytother Res 2008;22(2):228-237. 
15. Wei $Y$, Chen L, Chen J, Ge L and He RQ. Rapid glycation with D-ribose induces globular amyloid-like aggregations of BSA with high cytotoxicity to SH-SY5Y cells. BMC Cell Biology 2009; 10:10 doi: 10.1186/1471-2121-10-10.

16. Chompoo J, Upadhyay A, Kishimoto W, Makise T and Tawata S. Advance glycation end products inhibitors from Alpinia Zerumbet rhizomes. J Food chem 2011;129:709-715.

17. Electrophoresis Technical Handbook: [online] 2010. Available from: URL: http://sinapseinc.com/files/Handbook-Eletroforese. pdf

18. Meeprom A, Sompong W, Chan CB and Adisakwattana S. Isoferulic Acid, a New Anti-Glycation Agent, Inhibits Fructoseand Glucose-Mediated Protein Glycation in Vitro. Molecules 2013;18:6439-6454.

19. Ayatollahi SAM, Kobarfard F, Asgarpanah J and Choudhary MI.
Antiglycation activity of Otostegia persica (Burm.) Boiss. Afr $\mathrm{J}$ Biotechnology 2010;9(24):3645-3648.

20. Sauradipta B and Abhay C. In Vitro Study on Structural Alteration of Myoglobin by Methylglyoxal. Protein Journal. 2013;32(3): 216-222.

21. Ledesma-Osuna Al, Ramos-Clamont $\mathrm{G}$ and VázquezMoreno L. Characterization of bovine serum albumin glycated with glucose, galactose and lactose. Acta Biochim Pol 2008;55(3):491-497.

22. Gupta P, Nahata A and Dixit VK. An update of Murraya koenigii Spreng: a multifunctional Ayurvedic herb. J Chinese Integrative Medicine 2011;9(8):824-833.

23. Tembhurne SV and Sakarkar DM. Influence of Murraya koenigii on experimental model of diabetes and progression of neuropathic pain. Res Pharm Sci 2010;5(1):41-47.

Authors Contribution:

DCRW - Conducting experiments, data collection, literature search and manuscript preparation; HKIP - Concept and design of the study, obtaining grants, data collection, literature search, analysis and interpretation, manuscript preparation and critical revision of the manuscript.

Source of Support: University of Peradeniya Research Grants RG/2011/27/M and RG/2012/CG-1/38/M, Conflict of Interest: None declared. 